\title{
Expanding Indications of Primary Arthrodesis in Selected Individuals for Managing Complex Hindfoot Trauma During COVID-19 Pandemic
}

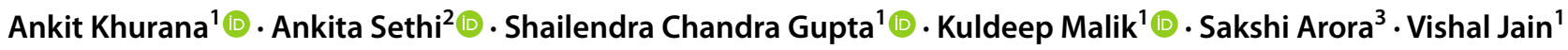

Received: 19 August 2021 / Accepted: 17 September 2021 / Published online: 15 October 2021

(c) Indian Orthopaedics Association 2021

\begin{abstract}
Background During the COVID-19 pandemic, public health measures to encourage social distancing have been implemented, including cancellation of outdoor activities, organized sports, and schools/colleges. Neglected hindfoot fractures have emerged as a consequence with increased frequency. Similarly, complex ankle and pilon fractures that require staged management, prolonged hospital stay, and soft-tissue care have emerged as a potential concern as prolonged exposure to healthcare setting adds to risk of acquiring as well as transmitting COVID-19 infection. The authors present their experience with expanding these indications for hindfoot arthrodesis as they encounter a greater number of neglected ankle and hindfoot trauma.

Methods This was a retrospective observational study of collected data from the trauma unit of our hospital. Inclusion criteria included all trauma classified by the AO/OTA as occurring at locations 43 , and who underwent subtalar and ankle arthrodesis. This included distal tibia, malleolar, talus, and calcaneus fractures. These patients were followed up to at least 6 months till complete fracture union.

Results A total of 18 patients underwent arthrodesis of either the ankle or subtalar joint between March and October 2020. Mean age of patients undergoing arthrodesis of the hindfoot was 69.2 years (43-84 years). Indications for the procedure included Displaced and comminuted intra-articular distal tibia fractures in elderly (6 patients), Malunited ankle fractures ( 2 patients), Neglected Ankle fractures managed conservatively (3 patients), Calcaneus fractures (5 patients), and neglected Talus body fracture ( 2 patients). All patients were followed up to at least 6 months and everyone went onto successful painless union between 3 and 6 months of the arthrodesis procedure without any significant complications.

Conclusion In summary, COVID-19 pandemic has led to a change in paradigm of trauma management and foot and ankle management is no different than other musculoskeletal trauma systems. The authors propose an expansion of indications for hindfoot arthrodesis in managing complex hindfoot trauma in pandemic situation.
\end{abstract}

Keywords Hindfoot $\cdot$ Arthrodesis $\cdot$ COVID-19 $\cdot$ Pandemic $\cdot$ Foot and ankle trauma

\section{Introduction}

During the COVID-19 pandemic, public health measures to encourage social distancing have been implemented, including cancellation of outdoor activities, organized sports, and

Ankit Khurana

ankit24388@gmail.com

1 Department of Orthopaedics, ESI Hospital Rohini, Delhi, India

2 Department of Obstetrics and Gynaecology, AIIMS, New Delhi, India

3 Department of Anaesthesia, ESI Hospital Rohini, Delhi, India closure of schools and colleges [1]. The severe disruptions caused by the SARS-CoV-2 coronavirus have necessitated a redistribution of resources to meet hospitals' current service needs during this pandemic. A resulting change in fracture epidemiology has emerged. Studies have highlighted this as an opportunity for simplified patient care during the pandemic [2].

Most traumatological presentations decreased in frequency over the course of the outbreak [3]. COVID-19 protocols have affected the epidemiology of foot and ankle trauma as well as how they are managed [4]. By limiting surgeries, unnecessary exposure and contamination were limited for both patients and healthcare workers [5]. However, this also results in delayed assessments for many patients, 
and ultimately adds to the healthcare burden, as some may not receive care until their disease is more advanced and potentially requiring hospital admission.

Neglected hindfoot fractures have emerged as a consequence with increased frequency. With increasing reduction acceptability so have malunited calcaneum and ankle fractures. Similarly, complex ankle and pilon fractures that require staged management, prolonged hospital stay, and soft-tissue care have emerged as a potential concern as prolonged exposure to healthcare setting adds to the risk of acquiring as well as transmitting COVID-19 infection.

Ankle arthrodesis is still a gold standard salvage procedure for the management of ankle arthritis [6]. Primary ankle arthrodesis combined with fracture reduction has emerged as an option for the severely comminuted tibial pilon fracture which restores acceptable function in a selected group of patients [7]. Similarly, subtalar arthrodesis is a highly effective option for patients with malunited calcaneum fractures [8]. The authors present their experience with expanding these indications for hindfoot arthrodesis as they encounter a greater number of neglected ankle and hindfoot trauma.

\section{Methods}

This was a retrospective observational study of collected data from the trauma unit of our hospital. The study was conducted under the auspices of a service evaluation; therefore, no ethical approval was required. All surgically treated foot and ankle trauma cases who underwent hindfoot arthrodesis admitted to the ESI hospital Rohini were included. These parameters were retrospectively analysed.

Inclusion criteria included all trauma classified by the AO/OTA as occurring at locations 43 , and who underwent subtalar and ankle arthrodesis. This included distal tibia, malleolar, talus, and calcaneus fractures. These patients were followed up for at least 6 months till complete fracture union. Charcot arthritis was ruled out in all cases using clinical evaluation and investigations and patients falling in the category of charcot arthropathy and not following inclusion criteria were excluded. The study was completed according to STROBE guidelines for observational studies [10].

\section{Results}

A total of 18 patients underwent arthrodesis of either the ankle or subtalar joint who met the above-mentioned inclusion criteria between March and October 2020. The mean age of patients undergoing arthrodesis of the hindfoot was 69.2 years (43-84 years). Indications for the procedure included Displaced and comminuted intra-articular distal tibia fractures in the elderly (6 patients), Malunited ankle fractures (2 patients), Neglected Ankle fractures managed conservatively ( 3 patients), Calcaneus fractures ( 5 patients), and neglected Talus body fracture (2 patients). All patients who presented with these indications were investigated on out-patient basis, advised limb elevation at home, and admitted within a week of presentation following medical fitness. The delay in presentation of included patients ranged from 7 to 98 days (Mean 55.6 days). While patients with fresh distal tibia intra-articular fractures in low-demand individuals were included and were considered for arthrodesis, while patients with neglected ankle fractures, neglected talus body fractures, and malunited ankle fractures were considered for arthrodesis. A similar approach was considered for Sanders type IV calcaneus fractures where the CT suggested difficult reduction of intra-articular calcaneus fractures with delayed presentation (over 3 weeks).

Hospital stay was kept to a minimum with patients staying only 3-10 days including admission a day before surgery (Mean stay 5.1 days). The stay was slightly delayed when the author believed the wound to show delayed healing in one case of talus fracture where the stay was 10 days. In remaining cases, patients were discharged within a maximum of 7 days from admission. All patients underwent an rtPCR for COVID-19 on the day of admission and a day prior to surgery as per hospital policy.

The approach for ankle arthrodesis was transfibular in all but one case where, due to excessive scar on the fibular side, a decision was taken to open the ankle joint from an anterior approach. Fixation devices ranged from cancellous screws to locking plates and tibio-talo-calcaneal nail (TTC nail) in one case. For subtalar arthrodesis, the approach was lateral extensile approach that was fresh and needed some degree of tuberosity reduction to attain heel height and reduce heel width prior to subtalar fixation. Where the fracture was sufficiently neglected that reduction was not needed and lateral wall exostosis was not significant enough to require exostectomy, a limited open sinus tarsi approach was chosen.

All patients were followed up to at least 6 months following primary arthrodesis procedure. All patients went onto successful painless union between 3 and 6 months of the arthrodesis procedure (Case examples Figs. 1, 2, 3 and 4). All patients were involved in the decision-making process of choosing either traditional protocol for managing their trauma which in most scenarios included span, scan, and plan. However, none of the patients chose the same owing to counselling and understanding the need for a shorter hospital stay with reliable results of arthrodesis procedure (Table 1). There are no complications including soft-tissue complications in any of the procedures and all wound healed within 2-3 weeks of surgery. 


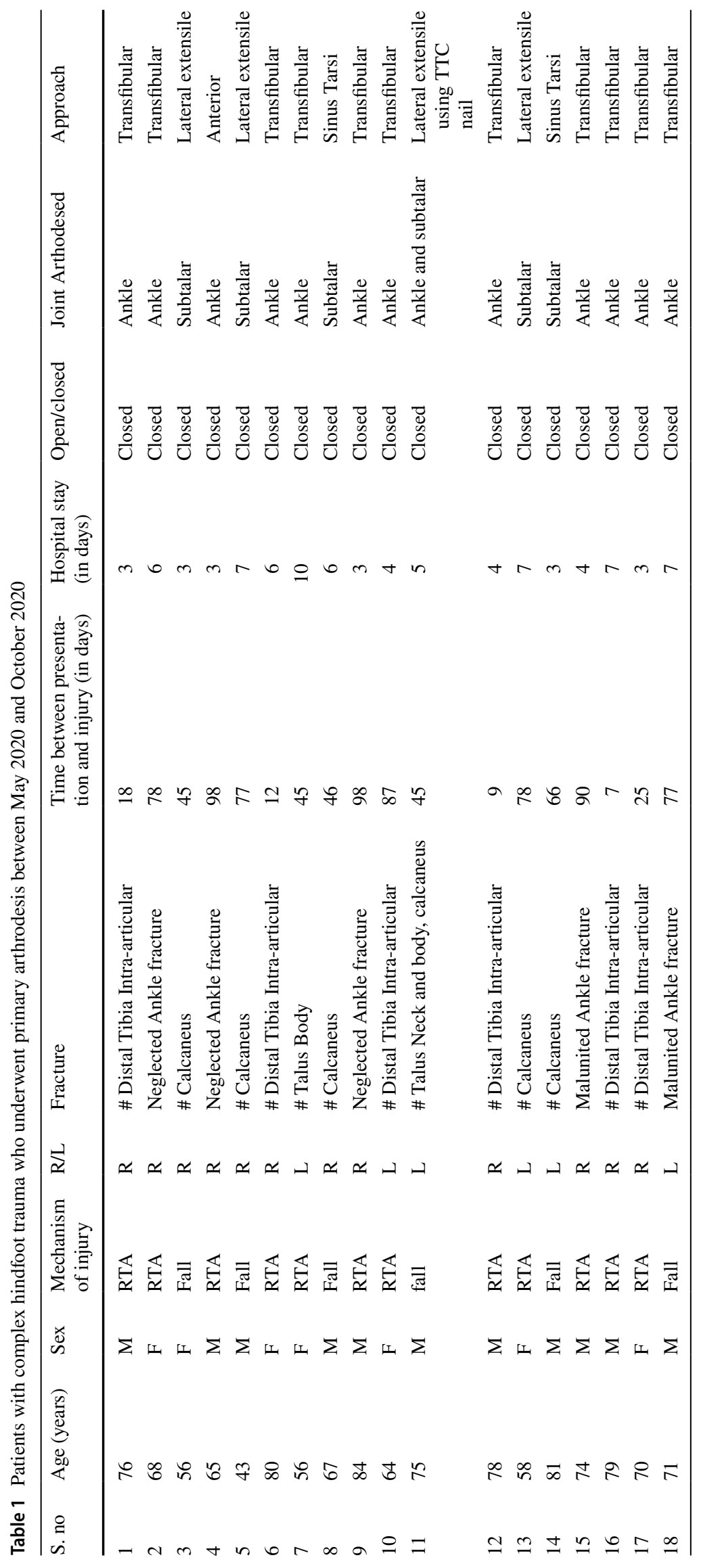




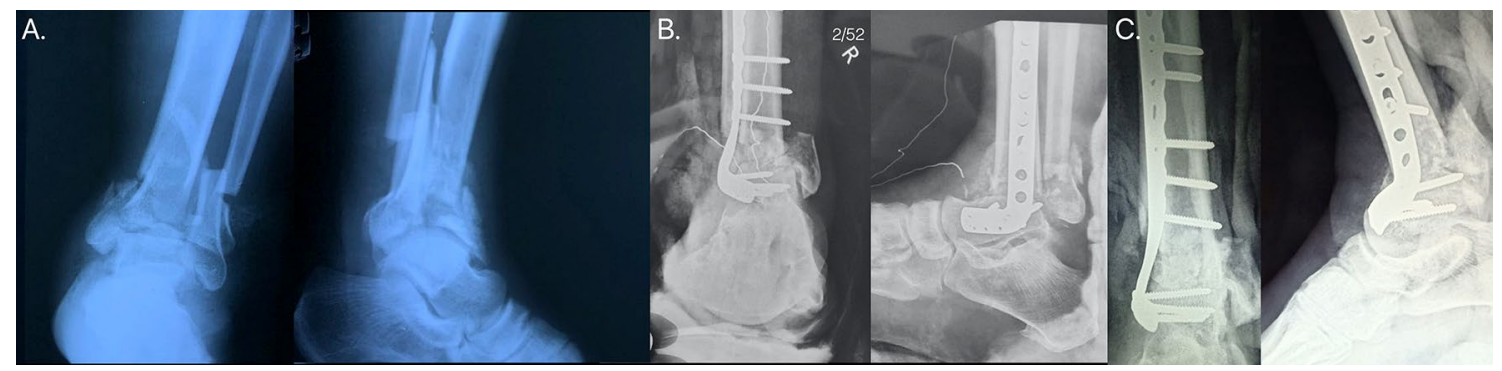

Fig. 1 Case example of a 76-year-old male with distal tibia intraarticular fracture presenting 18 days following trauma with poor skin condition (A). Patient underwent primary arthrodesis from a transfib- ular approach (B) and was followed up till 6 months when the patient had complete fusion of the ankle joint and a relatively normal gait $(\mathbf{C})$

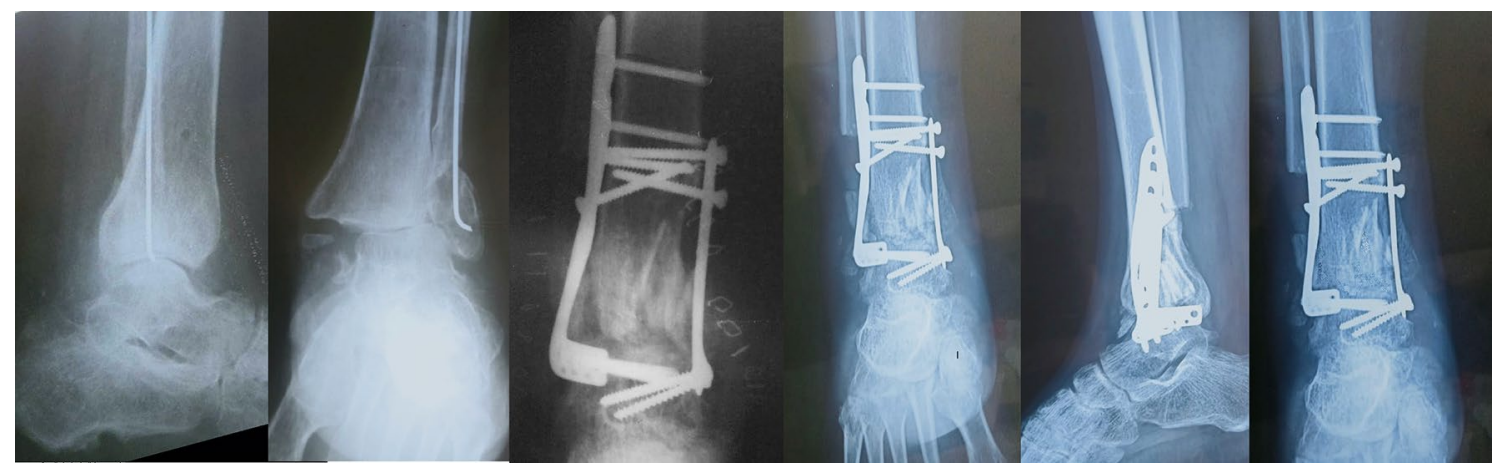

Fig. 2 Case example of an ankle fracture in a 68-year-old female managed initially by limited percutaneous fixation elsewhere but managed now with ankle arthrodesis and followed up till union of fusion site

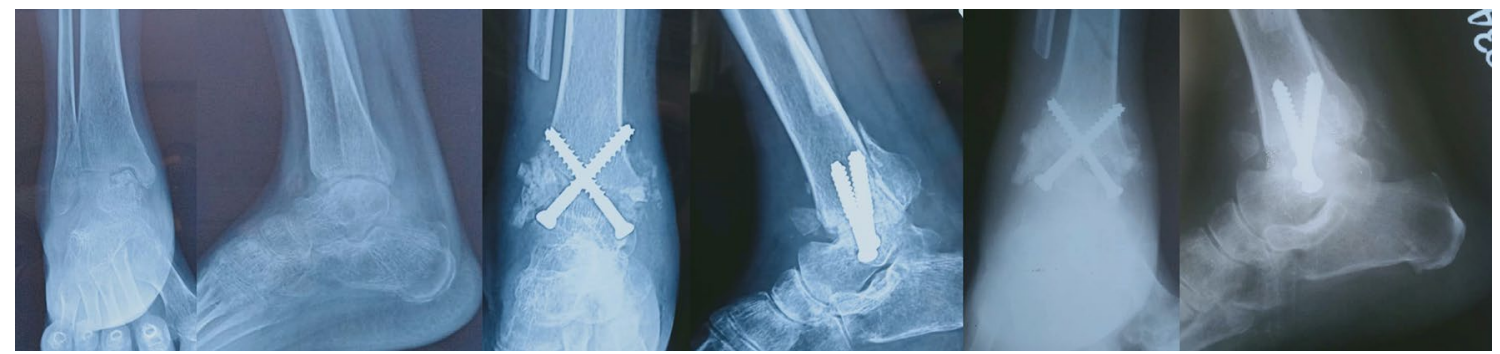

Fig. 3 Case example of a neglected talus body fracture in a 56-year-old female who presented with non-union and was subsequently managed with ankle arthrodesis and followed till union

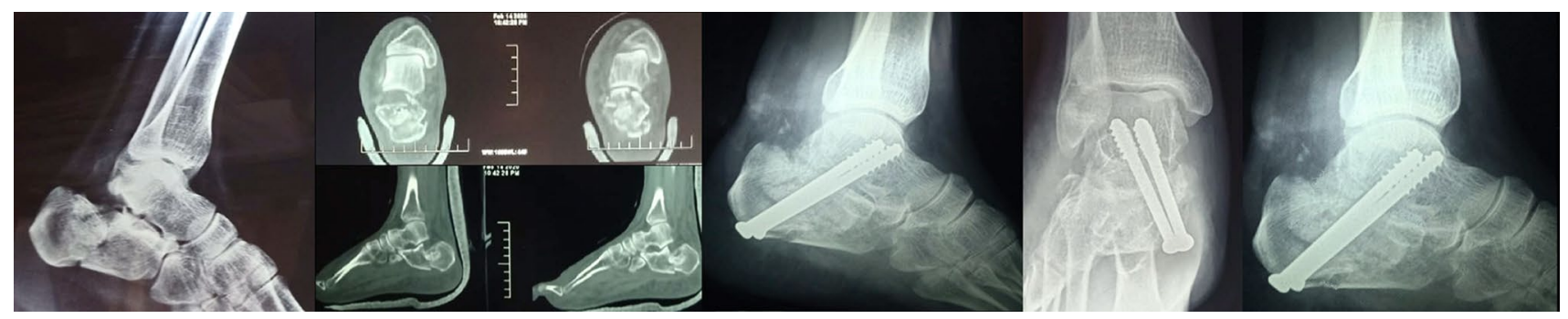

Fig. 4 Case example of displaced intra-articular Sanders IV calcaneus fracture managed with primary subtalar fusion along with bone grafting. After a follow-up of 3 months, the patient was able to weight bear with no ambulatory aid 


\section{Discussion}

The aim of management of non-COVID patients in the present pandemic scenario is to provide effective and reliable treatment options while keeping patients at minimal risk for contracting COVID infection and simultaneously keeping healthcare costs to a minimum. This also involves minimizing specialized care, thus reducing the financial burden on an already strained healthcare system. The approach must also keep operative time to a minimum and preferably limited to a single procedure rather than staged surgeries which will protect both the patient as well as the involved healthcare workers. Additionally, costs related to multiple and long procedures need to be cut down and the solutions must circumvent the crisis in theatres and limited anaesthetic support during the pandemic. This has been recommended by various trauma associations and local orthopaedic bodies in their published guidelines [9].

In keeping with these goals, the trauma surgeons in our institute decided to increase the threshold for offering primary arthrodesis in complex articular and peri-articular trauma of the hindfoot. From March to October 2020, 18 patients underwent subtalar and ankle arthrodesis at our institute owing to the shared vision of our trauma team of expanding the indications of this procedure. This leads to acceptable results and reliable union of arthrodesis site in all patients by a minimum of 6 month follow-up. Primary fusion of the hindfoot joints led to speeding up of the recovery process and decrease pain in the long term.

The concept of trauma management changed to providing acceptable outcomes to low-demand individuals while keeping their contact with the healthcare system to the minimum [10]. This was done by reducing the hospital stay, avoiding multiple surgeries, and providing a singlestage procedure with definitive outcomes [11]. This not only reduced chances of getting COVID but also reduced the burden on the healthcare system, anaesthesia team, and cut healthcare costs [12]. This was especially beneficial as the presentation was delayed in most cases $[13,14]$. Some cases also had a trial of conservative management before the presentation [14].

This strategy was used in patients with complex hindfoot trauma [15]. The demand of the patient was assessed based on occupation, activities of daily living, ambulatory status, and financial needs. The assessment was subjective with patients who were older, with limited ambulation, restricted to home with no occupational burden were chosen for primary hindfoot arthrodesis.

Tibial pilon fractures are complex injuries that are difficult to treat for even the most skilled orthopaedic trauma surgeons [16]. Traditionally, surgical treatment of distal tibial pilon fractures has been associated with increased rates of complications, namely non-union, infections, as well as rarely the need for amputation [5]. The current management of choice for tibial pilon fractures is staged management using the strategy of span, scan, and plan [17]. The mainstay of treatment today is still open reduction and internal fixation for the vast majority of pilon fractures as this provides direct visualization of the articular reduction and allows for direct reduction of metadiaphyseal segment. Although there are no definitive indications for primary arthrodesis, it has been used in cases in which reconstruction of the articular surface is not feasible, either due to the extensive articular comminution, articular cartilage damage, as well as in elderly patients who are poor candidates for multiple procedures with prolonged weight-bearing restrictions [18]. In a retrospective review of 20 patients treated with primary tibiotalar arthrodesis, Zelle et al. identified no wound complications or dehiscence with independent ambulation at the time of final follow-up and SF-36 scores similar to those historically reported for ORIF [19]. Similar recent studies assessing primary tibiotalar arthrodesis for complex pilon fractures have shown good outcomes with high union rates of the fusion and good functional outcomes with minimal soft-tissue complication rates [7, 20]. This formed the basis of our study as recent literature indicates acceptable outcomes and eliminates the need for multiple staged surgeries.

Ankle arthrodesis is considered a salvage operation for failed as well as neglected ankle fracture [21, 22]. Classic indications of ankle fusion in ankle fractures are diabetics with uncontrolled diabetes with a high risk of developing charcot arthropathy as well as patients with neglected fractures of the ankle with non-reconstructable malleoli or malunited ankle fractures in low-demand individuals as well as in patients with failed internal fixation of ankle fractures [23-25]. The authors expanded on these indications and performed ankle fusion in patients who presented late (over 12 weeks) or had undergone failed ankle fracture fixation. This strategy helps to minimize the incidence of operative failure, decreases duration of hospital stay, and prevents the risk of soft-tissue complications with open reduction and internal fixation [26].

The treatment of severely comminuted Displaced IntraArticular Calcaneal Fractures (DIACFs) remains controversial [27-31]. Although open reduction and internal fixation (ORIF) have become commonplace for displaced calcaneal fractures, more favourable outcomes have been reported in those patients with less-comminuted fracture patterns $[32,33]$. Primary subtalar arthrodesis for these fractures has gained acceptance in recent years [34]. In some instances, subtalar arthrodesis is eventually necessary to reduce the symptoms. Emerging evidence thus indicates that primary fusion is a reasonable strategy in 
managing these highly comminuted fractures [24, 35]. Recognising the near universal occurrence of subtalar joint arthrosis, regardless of the quality of the operative reduction in sander's type IV DIACFs, the authors performed primary subtalar arthrodesis to prevent repeat surgery and give the patient a suitable outcome with a single-stage procedure with the shorter hospital stay.

Similarly, the most common and best studied treatment of neglected fracture dislocation and avascular necrosis of talus is the tibiotalar arthrodesis using Blair's technique [36]. The risk of complications associated with talus fracture is far too many with a high rate of Avascular Necrosis (AVN) of the talus with the subsequent collapse. Additionally, the risk of secondary arthritis follows malunion, especially following delayed operative management [37]. A delay in surgical management magnifies these complications and making risk of AVN inevitable [21]. To avoid multiple surgeries and risks of complications, the straightforward technique is that of tibiotalar fusion in elderly patients presenting with old fracture. The authors chose this strategy for two patients; one of whom also had an associated calcaneus fracture and therefore underwent a TTC (Tibio-Talo-Calcaneal) fusion.

The strengths of this study are that this is the first case series reported for patients undergoing primary arthrodesis of complex hindfoot fracture during the pandemic which has dwelled upon the descriptive characteristics and associated complications of these injuries. Weaknesses included the small sample size of patients who were treated with primary arthrodesis. Second, this was a retrospective review, and multiple variables were dependent on appropriate documentation by medical providers in the chart, and clinical/functional outcomes were unable to be assessed.

In summary, COVID-19 pandemic has led to a change in the paradigm of trauma management and foot and ankle management is no different than other musculoskeletal trauma systems. The authors propose an expansion of indications for managing complex hindfoot trauma by primary arthrodesis of the involved hindfoot joints with the potential aim to reduce COVID-19 transmission and simultaneously minimize healthcare costs. Further prospective evaluation of this strategy during pandemic times would help to establish these protocols for managing hindfoot trauma in similar crisis.

\section{Declarations}

Conflict of Interest The authors state that there are no conflicts of interest.

Ethical Standard Statement This article does not contain any studies with human or animal subjects performed by the any of the authors.
Informed Consent For this type of study informed consent is not required.

\section{References}

1. The Lancet. (2020). India under COVID-19 lockdown. The Lancet, 395(10233), 1315.

2. Jain, V. K., Lal, H., Kumar Patralekh, M., \& Vaishya, R. (2020). Fracture management during COVID-19 pandemic: A systematic review. Journal of Clinical Orthopaedics and Trauma, 11(Suppl 4), S431-S441.

3. Shah, R., Ahad, A., Faizi, M., \& Mangwani, J. (2021). Foot and ankle trauma management during the COVID-19 pandemic: Experiences from a major trauma unit. Journal of Clinical Orthopaedics and Trauma., 16, 285-291.

4. Stringer, H., Molloy, A., Craven, J., Moorehead, J., Santini, A., \& Mason, L. (2021). The impact of COVID-19 on foot and ankle surgery in a major trauma centre. The Foot, 46, 101772.

5. Haut, E. R., Leeds, I. L., \& Livingston, D. H. (2020). The effect on trauma care secondary to the COVID-19 pandemic: Collateral damage from diversion of resources. Annals of Surgery, 272(3), e204-e207.

6. Gowda, B. N., \& Kumar, J. M. (2012). Outcome of ankle arthrodesis in posttraumatic arthritis. Indian Journal of Orthopaedics, 46(3), 317-320.

7. Beaman, D. N., \& Gellman, R. (2014). Fracture reduction and primary ankle arthrodesis: A reliable approach for severely comminuted tibial pilon fracture. Clinical Orthopaedics and Related Research, 472(12), 3823-3834.

8. Jackson, J. B., Jacobson, L., Banerjee, R., \& Nickisch, F. (2015). Distraction subtalar arthrodesis. Foot and Ankle Clinics, 20(2), 335-351.

9. Indian Orthopaedic Assocation [Internet]. (2021). https://www. ioaindia.org/COVID-19IOAguidelines.pdf. Accessed 16 May 2021.

10. Sawhney, C., Singh, Y., Jain, K., Sawhney, R., \& Trikha, A. (2020). Trauma care and COVID-19 pandemic. Journal of Anaesthesiology Clinical Pharmacology, 36(Suppl 1), S115-S120.

11. Padilla-Rojas, L. G., López-Cervantes, R. E., López-Almejo, L., Gutiérrez-Mendoza, I., Amadei-Enghelmayer, R. E., Pesciallo, C. A., et al. (2021). Orthopaedic trauma care during the COVID-19 Pandemic: The Latin American perspective. OTA International, 4(1S), e114.

12. Neradi, D., Hooda, A., Shetty, A., Kumar, D., Salaria, A. K., \& Goni, V. (2020). Management of orthopaedic patients during COVID-19 Pandemic in India: A guide. Indian Journal of Orthopaedics, 54(3), 402-407.

13. Khak, M., Manafi-Rasi, A., Oryadi Zanjani, L., \& Nabian, M. H. (2020). Orthopedic trauma surgeries in COVID-19 pandemic: A trauma management algorithm. Archives of Bone and Joint Surgery. https://doi.org/10.22038/abjs.2020.47741.2344

14. Jain, V. K., \& Vaishya, R. (2020). COVID-19 and orthopaedic surgeons: The Indian scenario. Tropical Doctor, 50(2), 108-110.

15. Chadwick, P., Ambrose, L., Barrow, R., \& Fox, M. (2020). A commentary on podiatry during the Covid-19 pandemic: Podiatry during the Covid-19 pandemic. Journal of Foot and Ankle Research, 13(1), 63.

16. Dillin, L., \& Slabaugh, P. (1986). Delayed wound healing infection, and nonunion following open reduction and internal fixation of tibial plafond fractures. The Journal of Trauma, 26(12), 1116-1119.

17. Carter, T. H., Duckworth, A. D., Oliver, W. M., Molyneux, S. G., Amin, A. K., \& White, T. O. (2019). Open reduction and internal 
fixation of distal tibial pilon fractures. JBJS Essential Surgical Techniques, 9(3), e29.

18. Bear, J., Rollick, N., \& Helfet, D. (2018). Evolution in management of Tibial pilon fractures. Current Reviews in Musculoskeletal Medicine, 11(4), 537-545.

19. Zelle, B. A., Gruen, G. S., McMillen, R. L., \& Dahl, J. D. (2014). Primary arthrodesis of the tibiotalar joint in severely comminuted high-energy pilon fractures. The Journal of Bone and Joint Surgery, 96(11), e91.

20. Ho, B., \& Ketz, J. (2017). Primary arthrodesis for tibial pilon fractures. Foot and Ankle Clinics, 22(1), 147-161.

21. Cheng, Y. M., Huang, P. J., Chen, S. K., Tien, Y. C., Lin, S. Y., Chen, L. H., et al. (1997). Salvage operation for neglected ankle fractures. Kaohsiung Journal of Medical Sciences, 13(10), $618-625$

22. Thomason, K., Ramesh, A., McGoldrick, N., Cove, R., Walsh, J. C., \& Stephens, M. M. (2014). Primary Ankle arthrodesis for neglected open Weber B ankle fracture dislocation. The Journal of Foot and Ankle Surgery, 53(4), 446-448.

23. Grote, C. W., Tucker, W., Stumpff, K., Birt, M. C., \& Horton, G. A. (2020). Primary arthrodesis for diabetic ankle fractures. Foot and Ankle Orthopaedics, 5(1), 247301142090884.

24. Wallace, S. J., Liskutin, T. E., Schiff, A. P., \& Pinzur, M. S. (2020). Ankle fusion following failed initial treatment of complex ankle fractures in neuropathic diabetics. Foot and Ankle Surgery, 26(2), 189-192.

25. Henderson, W. B., \& Lau, J. T. C. (2006). Reconstruction of failed ankle fractures. Foot and Ankle Clinics, 11(1), 51-60.

26. Mangwani, J., Mehta, S., Rees, K., \& Cutler, L. (2014). Understanding risks and complications in the management of ankle fractures. Indian Journal of Orthopaedics, 48(5), 445.

27. Khurana, A., Dhillon, M. S., Prabhakar, S., \& John, R. (2017). Outcome evaluation of minimally invasive surgery versus extensile lateral approach in management of displaced intra-articular calcaneal fractures: A randomised control trial. Foot (Edinburgh, Scotland), 31, 23-30.

28. Basile, A. (2010). Operative versus nonoperative treatment of displaced intra-articular calcaneal fractures in elderly patients. The Journal of Foot and Ankle Surgery, 49(1), 25-32.
29. Gaskill, T., Schweitzer, K., \& Nunley, J. (2010). Comparison of surgical outcomes of intra-articular calcaneal fractures by age. The Journal of Bone and Joint Surgery-American Volume, 92(18), 2884-2889.

30. Buckley, R. E. (2009). What is the best treatment for displaced intra-articular calcaneal fractures? Evidence-based orthopaedics (pp. 498-501). Elsevier.

31. Buckley, R., Tough, S., Mccormack, R., Pate, G., Leighton, R., Petrie, D., et al. (2002t). Operative compared with nonoperative treatment of displaced intra-articular calcaneal fractures: a prospective, randomized, controlled multicenter trial. The Journal of Bone and Joint Surgery-American Volume, 84(10), 1733-1744.

32. Bozkurt, M., Kentel, B. B., Yavuzer, G., Öçgüder, A., Heycan, C., \& Tonuk, E. (2004). Functional evaluation of intra-articular severely comminuted fractures of the calcaneus with gait analysis. The Journal of Foot and Ankle Surgery, 43(6), 374-379.

33. Csizy, M., Buckley, R., Tough, S., Leighton, R., Smith, J., McCormack, R., et al. (2003). Displaced Intra-articular calcaneal fractures: Variables predicting late subtalar fusion. Journal of Orthopaedic Trauma, 17(2), 106-112.

34. Schepers, T. (2012). The primary arthrodesis for severely comminuted intra-articular fractures of the calcaneus: A systematic review. Foot and Ankle Surgery, 18(2), 84-88.

35. Holm, J. L., Laxson, S. E., \& Schuberth, J. M. (2015). Primary subtalar joint arthrodesis for comminuted fractures of the calcaneus. The Journal of Foot and Ankle Surgery, 54(1), 61-65.

36. Singh, J. (2008). Tibiotalar arthrodesis for injuries of the talus. Indian Journal of Orthopaedics, 42(1), 87-90.

37. Jordan, R. K., Bafna, K. R., Liu, J., \& Ebraheim, N. A. (2017). Complications of Talar neck fractures by Hawkins classification: a systematic review. The Journal of Foot and Ankle Surgery, 56(4), 817-821.

Publisher's Note Springer Nature remains neutral with regard to jurisdictional claims in published maps and institutional affiliations. 